\title{
Nanocomposite of silk fibroin nanofiber and montmorillonite: fabrication and morphology
}

Yuki Kishimoto ${ }^{\mathrm{a}}$, Fuyu Ito ${ }^{\mathrm{b}}$, Hisanao Usami ${ }^{\mathrm{a}}$, Eiji Togawa ${ }^{\mathrm{c}}$, Masuhiro Tsukada $^{\mathrm{a}}$, Hideaki Morikawa ${ }^{\mathrm{a}}$,

Shigeru Yamanaka $a^{\mathrm{a}, *}$

${ }^{\mathrm{a}}$ Faculty of Textile Science and Technology, Shinshu University, 3-15-1 Tokida, Ueda, Nagano 386-8567,

Japan

${ }^{\mathrm{b} S a t e l l i t e ~ V e n t u r e ~ B u s i n e s s ~ L a b o r a t o r y, ~ S h i n s h u ~ U n i v e r s i t y, ~ 3-15-1 ~ T o k i d a, ~ U e d a, ~ N a g a n o ~ 386-8567, ~}$

Japan

${ }^{\mathrm{c}}$ Forestry and Forest Products Research Institute, 1 Matsunosato, Tsukuba 305-8687, Japan

\section{Corresponding author}

Shigeru Yamanaka

Phone: +919072140529

E-mail: s.y@jcom.home.ne.jp 


\section{ABSTRACT}

The purpose of our research is creating a new nanocomposite material. Generally silk fibroin (SF) is regarded as a promising base material for biomedical uses. The incorporation of montmorillonite (MMT) into SF fibers would improve physical properties of the SF fibers. We investigated a new method of combining electospun SF with MMT. Specifically, electrospun silk nanofibers were treated with methanol and dipped in a MMT suspension. We could obtain a nanosheet composite of silk nanofibers and MMT. Their ultrastructures were successfully visualized by high resolution transmission electron microscopy. This compound was comprised of individual silk nanofibers surrounded by thin layers of MMT, each with a thickness of about $1.2 \mathrm{~nm}$. This structure was confirmed by elemental analysis. We also performed IR, NMR and X-ray diffraction analyses in conjunction with morphological data.

Conclusively we obtained a new composite of silk nanofiber and MMT, which has never been reported. . Using this unique nanocomposite biological tests of its application for a scaffold for tissue engineering are under way.

\section{Keywords}

silk fibroin, electrospun nanofiber, montmorillonite, organic/inorganic nanocomposite, transmission electron microscopy 
In recent years, nano composites have gained much attention because of conspicuous change in properties, such as large surface, strength, high surface reactivity. ${ }^{1}$ Silkfibroin (SF) is a protein that has high chemical reactivity and mechanical property. ${ }^{2}$ In this study, we used electrospun SF to combine silica compound of MMT to produce silk/silica materials. The silk/silica was reported to be produced by combining SF with silica particles and used as osteoinductive composite for bone generation. ${ }^{3}$ Another example of silk/silica complex was prepared by sol-gel crosslinking process and the silica particles were bound to the fibroin, thermal properties being improved. ${ }^{4}$

This communication deals with our research in creating a new SF/MMT nanocomposite in a simple method. The product, however, had a unique nanostructure in which nano SF and thin MMT are combined. We analyzed this nanostructure by electronmicroscopes and disclosed its details.

Cocoons of Bombix mori silkworm silk were used for the preparation of SF nanofiber. SF was extracted from silk composed of fibroin and sericine according to the method described. ${ }^{5}$ Cocoons were sliced into four pieces, being suspended in a boiled $2.5(\mathrm{w} / \mathrm{v}) \%$ sodium carbonate solution, followed by boiling for $30 \mathrm{~min}$ to obtain scoured cocoon. The scoured cocoons were washed in running tap water for two days. The washed specimens were air-dried and dried in an oven at $105{ }^{\circ} \mathrm{C}$ for $90 \mathrm{~min}$. SF fibers were dissolved in a $9 \mathrm{M}$ lithium bromide solution at $55-60{ }^{\circ} \mathrm{C}$, and then dialyzed through cellulose membrane against distilled water. The aqueous fibroin solution was freeze-dried to give dry SF powder (regenerated SF). The regenerated SF powder was dissolved in trifluoro acetic acid (TFA), to prepare $8 \mathrm{wt} \%$, 10wt\%, $12 \mathrm{wt} \%$ SF/TFA solution. 
The electrospinning apparatus used was of Kato Tech Company (Kyoto, Japan), operated at room temperature. Procedure was basically accorded with the paper6 dealing with fabrication of silk sericine nanofibers from our laboratory. Electrospinning produced a non-woven sheet of randomly arranged nanofibers with nanometer scale diameters. In an electrospinning, an electric voltage (20-30 $\mathrm{kV})$ was applied to droplets of SF/TFA solution. The electrospun nanofibers were collected on a collector of wax paper, which was placed at a distance of $15 \mathrm{~cm}$ from the syringe. During SF/TFA solution is jetted in the air most of TFA is evaporated. The morphologies of the SF nanofiber were observed using scanning electron microscopy (SEM: JSM-6010LA; JEOL Ltd, Tokyo, Japan) at $10 \mathrm{kV}$ after being sputter-coated with platinum. SF nanofiber morphologies were influenced by electrospinning conditions. ${ }^{7}$ In electrospinning processes, polymer solution and applied voltages are important factors. Figure 1shows images of the specimen with applied voltage at $20 \mathrm{kV}$. When the concentration of SF/TFA was $8 \mathrm{wt} \%$, the beads appeared(Figure 1(a)).,. On the otherhand, at 10wt\% SF/TFA solutionthe beads disappeared (Figure 1(b)). When the applied voltage was raised $30 \mathrm{kV}$, almost the same results were obtained (data not shown).

Operating at $20 \mathrm{kV}$, when the concentration of SF/TFA solution were $8 \mathrm{wt} \%$, $10 \mathrm{wt} \%$ and $12 \mathrm{wt} \%$, fibers' diameter obtained were $118 \mathrm{~nm}, 214 \mathrm{~nm}$ and $399 \mathrm{~nm}$ respectively. At $30 \mathrm{kV}$, fibers diameter obtained were $391 \mathrm{~nm}, 398 \mathrm{~nm}$ and $559 \mathrm{~nm}$ in a concentration of $8 \mathrm{wt} \%, 10 \mathrm{wt} \%$ and $12 \mathrm{wt} \%$ respectively. In this study, we selected the condition for the fiber formation at $10 \mathrm{wt} \%$ and $20 \mathrm{kV}$, conditions which gave fine fibers of about 200nm in diameter, without beads (Figure 1 (b)).

To make a composite of SF nanofiber and MMT, SF nanofibers were tried to 
immerse in a pure methanol (WAKO Ltd.) solution for $15 \mathrm{~min}$., and the suspension was dried at room temperature for 24 hours. Through methanol treatment SF nanofibers were transformable from soluble to insoluble form of B-sheets conformation. ${ }^{8}$ The conformation of as-spun SF nanofiber is known to be a-helix in structure, on the other hand, methanol-treated SF nanofiber is known to be B-sheet in structure. ${ }^{8}$ FT-IR (FT-IR, IR Prestige-21; Shimadzu, Ltd, Tokyo, Japan) spectroscopy has been applied to study the molecular conformation of SF nanofiber (Figure 2). We examined the FT-IR spectra in the amide I and II regions. The as-spun SF was characterized by absorption bands at $1651 \mathrm{~cm}^{-1}$ (amid I) and $1528 \mathrm{~cm}^{-1}$ (amid II), attributed to the a-conformation. ${ }^{8}$ On the other hand, methanol treated SF nanofiber showed B-sheet conformation at $1623 \mathrm{~cm}^{-1}$ (amid I) and $1515 \mathrm{~cm}^{-1}$ (amid II). 8

To develop new materials, we interacted with SF and MMT. MMT used was purchased from Kunimine Industry Co. Ltd., Japan (Kunipia F). A clay of montmorillonite (MMT) is one of the most commonly used layered silicates, with a formula, $\mathrm{Mx}\left(\mathrm{Al}_{4}{ }^{-x M g x}\right) \mathrm{Si}_{8} \mathrm{O}_{20}$ in crystal lattice of octahedral sheet. ${ }^{9}$ The $\mathrm{SF}$ nanofibers were dipped in a MMT aqueous suspension at concentrations of $0.1 \mathrm{wt} \%, 0.5 \mathrm{wt} \%$ and $1 \mathrm{wt} \%$, the product being dried at room temperature for 12 hours to produce SF/MMT composites. The morphology of the SF/MMT composites were observed using field emission SEM (FESEM: SU8000 and SU8040; Hitachi HTA, Inc, Tokyo, Japan) at $20 \mathrm{kV}$ and $0.8 \mathrm{kV}$. Cross-section surface was Ar-ion milled using Hitachi IM4000 and was sputter-coated with platinum. Figure 3(a) shows that the film obtained SF nanofiberwhich is apparently joined with MMT (the concentration of MMT aqueous suspension was 
$0.5 \mathrm{wt} \%$ ) At $0.1 \mathrm{wt} \%$ and $1 \mathrm{wt} \%$ the products were the basically same (data not shown). Compared with Figure 1, it is obvious that the morphology of nanofibers have been unaltered. The nano SF/MMT composites in a sheet form were sliced to view cross-sectional figure (Figure 3(b)). Fibrous structures of nano SF are intertwisted in a sheet about $2.5 \mu \mathrm{m}$ thickness. SF nanofiber is almost circular.

Moreover, transmission electron microscopy (TEM: JEM-2100F, JEOL Ltd, Tokyo, Japan) analyses were done to observe detailed structures of this specimen (Figure 4). As shown by circles the thin films were formed. Energy dispersive spectroscopic (EDS) analysis was performed to investigate the entity of the thin films and judging from the composition the film was identified as MMT (data not shown).

The next problem is how the MMT is correlated with SF nanofiber. For this purpose the composite was observed by TEM (JEM-2800; JEOL Ltd, Tokyo, Japan) at $200 \mathrm{kV}$ and analyzed by EDS in detail. EDS data was collected on a JEOL JED-2300T EDS system combined with JEOL JEM-2100F. Ultrathin sections $(80 \mathrm{~nm})$ were embedded in Epon and cut on a Leica Ultracut UCT. These photos demonstrate the single SF nanofiber is surrounded by thin MMT layer.

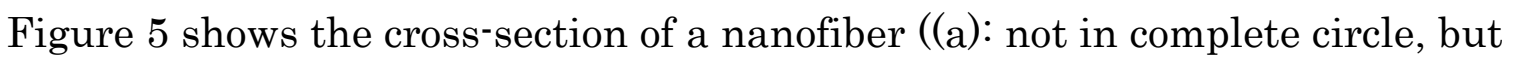
distorted because probably of artifact during slicing). In Figure 5 (b) enlarged to $20 \mathrm{k}$ magnification, layered construction was observed. To confirm a distributed composition of MMT. TEM-EDS mapping examinations were carried out as shown in Figure 5(c)-(h). The EDS mapping results indicate that $\mathrm{C}, \mathrm{O}, \mathrm{Al}, \mathrm{Si}$ and Mg atoms, which are MMT constituent elements, are uniformly distributed on the surface of SF nanofiber. Thus, SF/ MMT composites have been successfully 
synthesized via dipping in a MMT suspension. From above results the detailed morphology of SF/MMT nanocomposite was disclosed clearly. This is the first success of creating a new composite in which the SFs are surrounded by layers of MMT in thickness of around $1.2 \mathrm{~nm}$.

Previous silk/MMT clay or silk/clay composites reported were MMTs were dispersed in silk protein ${ }^{10}$ or silica particles were incorporated into film form silk or bound to SF. ${ }^{3,4}$ These are quite different from above our mentioned new silk/MMT nanocomposite.

The mechanistic analyses of interaction between SF nanofiber and MMT remain to be performed.

Generally $\mathrm{SF}$ is a candidate for the biomedical like tissue culture (tissue scaffold) and composite industries. Incorporation of clay into SF would improve tensile strength and heat tolerance. This material is unique in the point that each SF nanofiber is surrounded or protected by clay (MMT). Based on new composite nano structure we will pursue not only new functional properties or new applications as future research items.

In this study, we produced silk nanofiber/montmorillonite composite material. We analyzed the new material focusing on detailed ultrastructure of the composite. MMT layers in $1.2 \mathrm{~nm}$ thickness were bound with spun silk nanofiber. This is the first report of a unique structure of SF covered with nanolayers of MMT. Such covering of individual nanosilk with MMT clay is speculated to open a new field of material science. Moreover such composites are created by just dipping of SF nanofiber in a MMT suspension. 


\section{ACKNOWLEDGEMENTS}

We are grateful to Dr. M. Tsukada (Shinshu University) and Dr. Y. Murakami (Shinshu University) for supply of cocoon and, helpful advice and encouragement respectively.

We are very grateful to JEOL Ltd. and Hitachi HT Co. Ltd. who kindly took the eletronmicrograph of the nanocomposite

\section{REFERECES}

(1) Bhardwaj, N.; Kundu, S. C. Electrospinning: A fascinating fiber fabrication technique. Biotechnology Advances 2010, 28, 325-347.

(2) Vepari, C.; Kaplan, D. L. Silk as a biomaterial. Prog. Polym. Sci. 2007, 32, 991-1007.

(3) Mieszawska, A. J.; Fourligas, N.; Georgakoudi, I.; Ouhib, N. M.; Belton, D. J.; Perry, C. C.; Kaplan, D. L. Osteoinductive silk-silica composite biomaterial for bone regeration. Biomaterial 2010, 31, 8902-8910.

(4) Hou, A.;Chen, H. Preparation and characterization of silk/silica hybrid biomaterials by sol-gel crosslinking process. Material science and engineering $B$ 2010, 167, 124-128.

(5) Chen, C.; Chuanbo, C.; Xilan, M.; Yin, T.; Hesun, Z. Preparation of non-woven mats from all-aqueous silk fibroin with electrospinning method. Polymer 2006, 47, 6322-6327.

(6) Zhang, X.; Khan, M. M. R.; Yamamoto, T.; Tsukada, M.; Morikawa, H. Fabrication of silk sericin nanofibers from a silk sericin-hope cocoon with electrospinnig method. Int. J. Biol. Macromolecules 2012, 50,337-347.

(7) Sukigara, S.; Gandhi, M.; Ayutsede, J.; Micklus, M.; Ko, F. Regeneration of Bombyx mori silk by electrospinning-Part1: processing parameters and geometric properties. Polymer 2003, 44, 5721-5727.

(8) Kim, S. H.; Nam, Y. S.; Lee, T. S.; Park, W. H. Silk fibroin nanofiber. Electrospinning, properties, and structure. Polymer Journal 2003, 35(2), 185-190. (9) Mieszawska, A. J.; Llamas, J. G.; Vaiana, C. A.; Kadakia, M. P.; Naik, R. R.; Kaplan, D. L. Clay enriched silk biomaterials for bone formation. Acta Biomaterialia 2011, 7, 3036-3041.

(10) Dang, Q.; Lu, S.; Yu, S.; Sun, P.; Yuan, Z. Silk fibroin/montmorilonite nanocomposites: effect of $\mathrm{pH}$ on the conformational transition and clay dispersion. Biomacromolecules 2010, 11(7), 1796-1801. 
Fig. 1
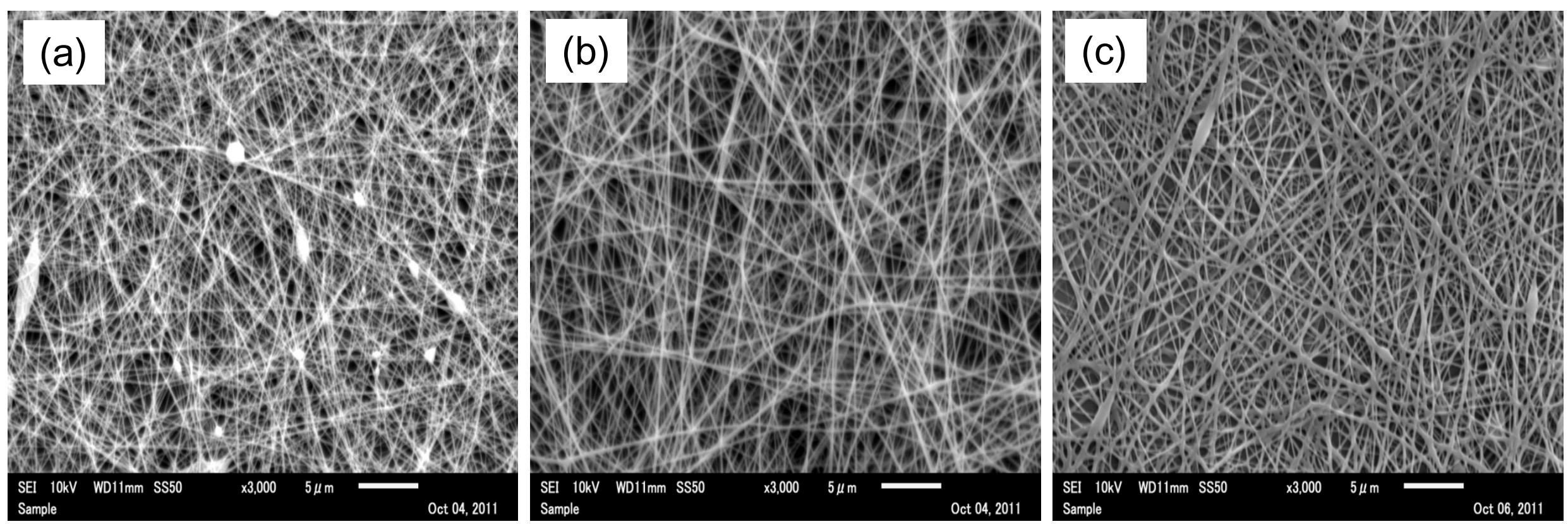

Nanocomposite of silk fibroin nanofiber and montmorillonite: fabrication and morphology

Yuki Kishimoto, Fuyu Ito, Hisanao Usami, Eiji Togawa, Masuhiro Tsukada, Hideaki Morikawa, Shigeru Yamanaka 
Fig. 2

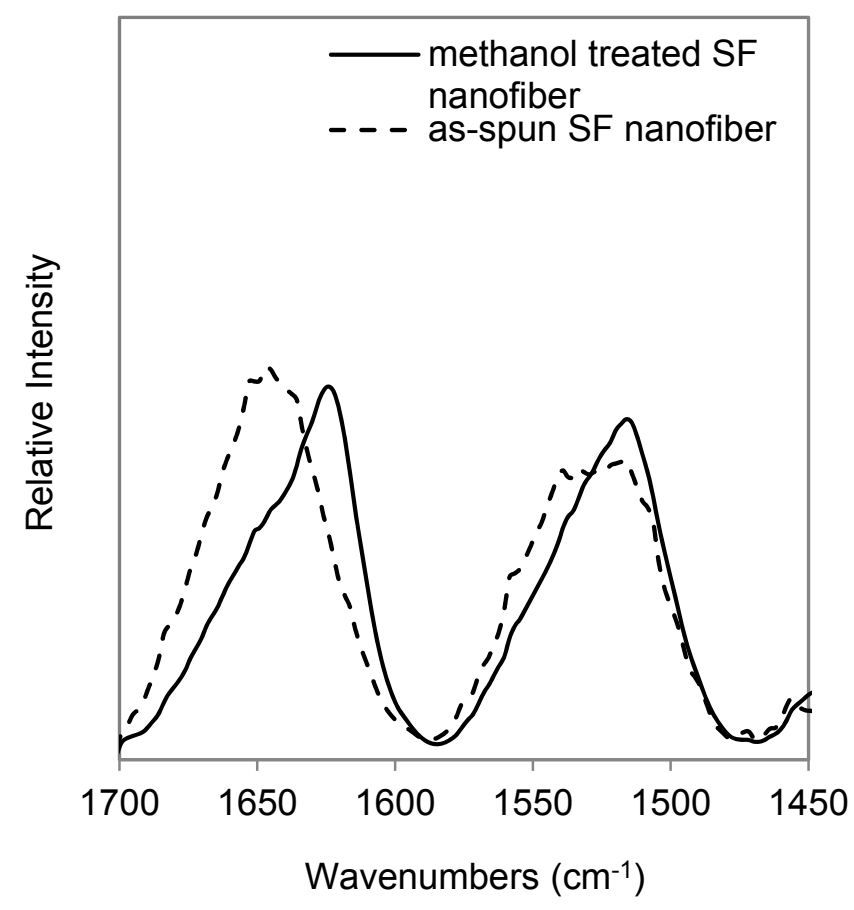

Nanocomposite of silk fibroin nanofiber and montmorillonite: fabrication and morphology

Yuki Kishimoto, Fuyu Ito, Hisanao Usami, Eiji Togawa, Masuhiro Tsukada, Hideaki Morikawa, Shigeru Yamanaka 
Fig. 3
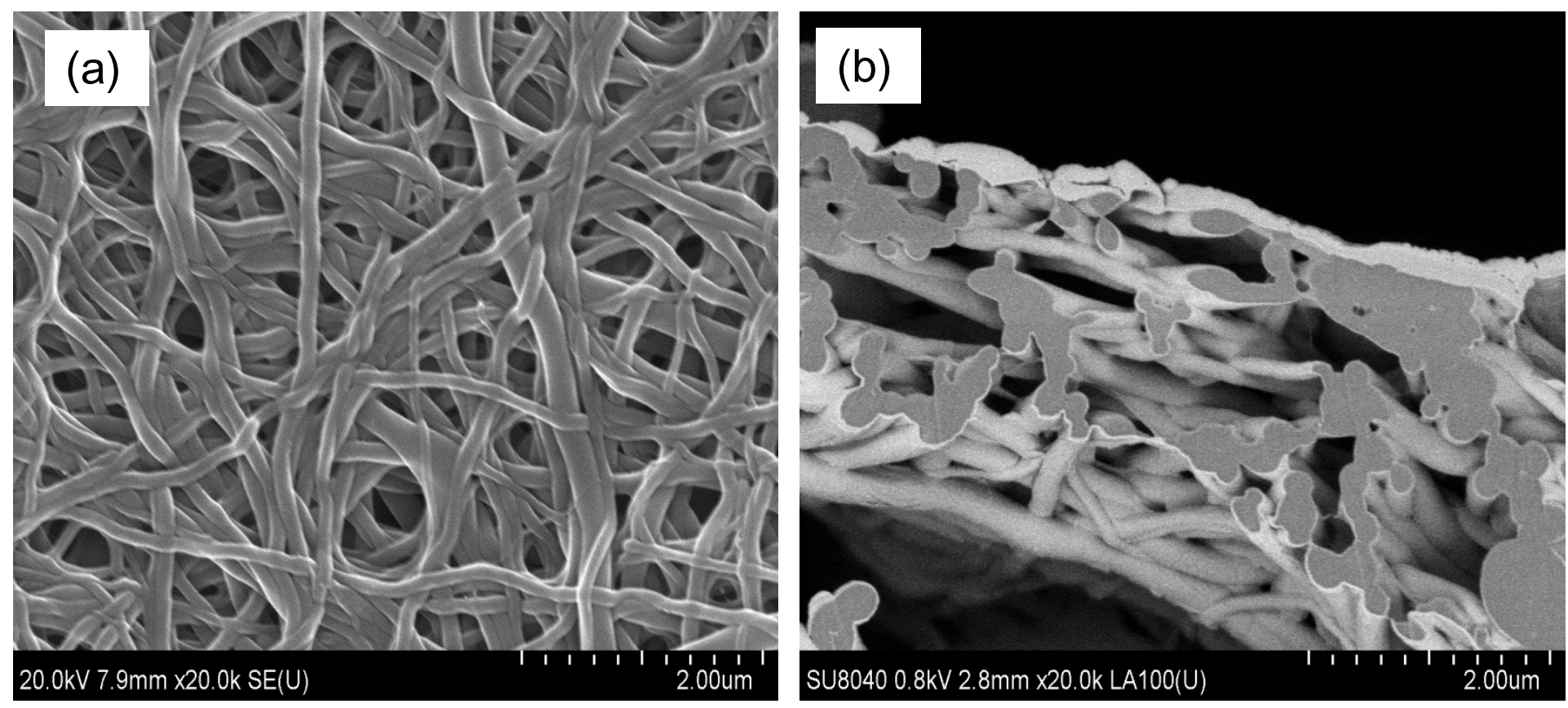

Nanocomposite of silk fibroin nanofiber and montmorillonite: fabrication and morphology

Yuki Kishimoto, Fuyu Ito, Hisanao Usami, Eiji Togawa, Masuhiro Tsukada, Hideaki Morikawa, Shigeru Yamanaka 
Fig. 4

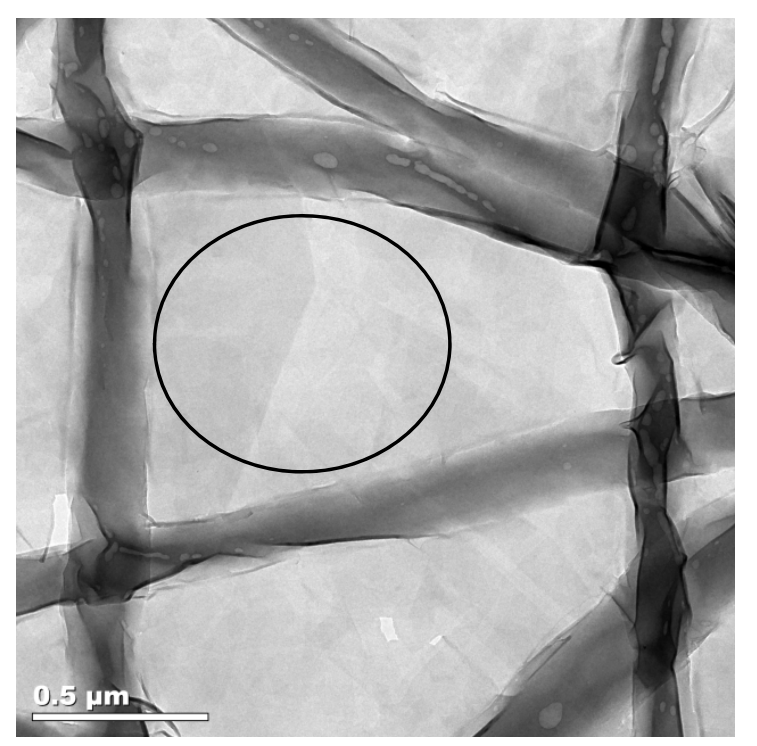

Nanocomposite of silk fibroin nanofiber and montmorillonite: fabrication and morphology

Yuki Kishimoto, Fuyu Ito, Hisanao Usami, Eiji Togawa, Masuhiro Tsukada, Hideaki Morikawa, Shigeru Yamanaka 
Fig. 5
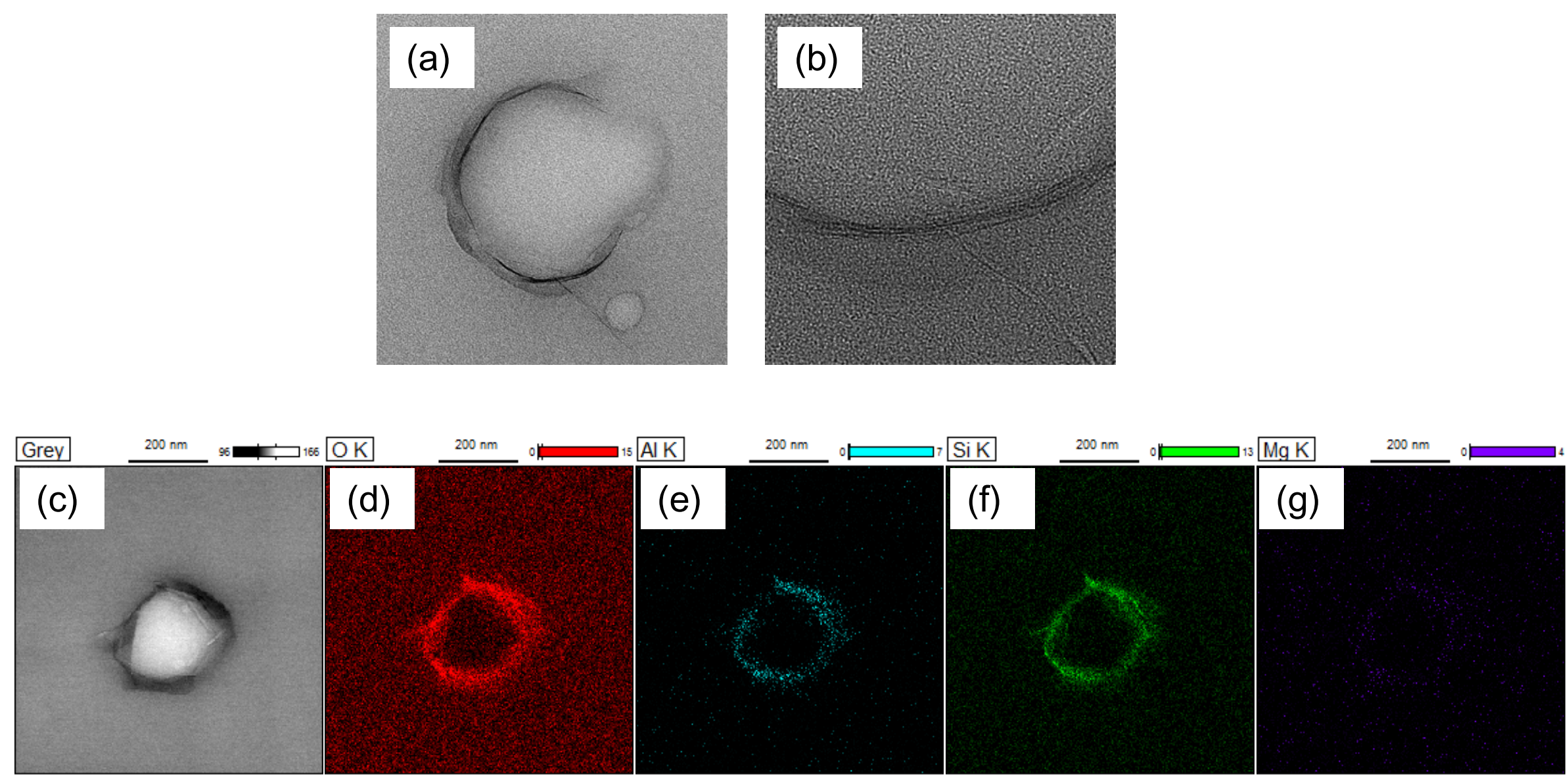

Nanocomposite of silk fibroin nanofiber and montmorillonite: fabrication and morphology

Yuki Kishimoto, Fuyu Ito, Hisanao Usami, Eiji Togawa, Masuhiro Tsukada, Hideaki Morikawa, Shigeru Yamanaka 
Fig. 6

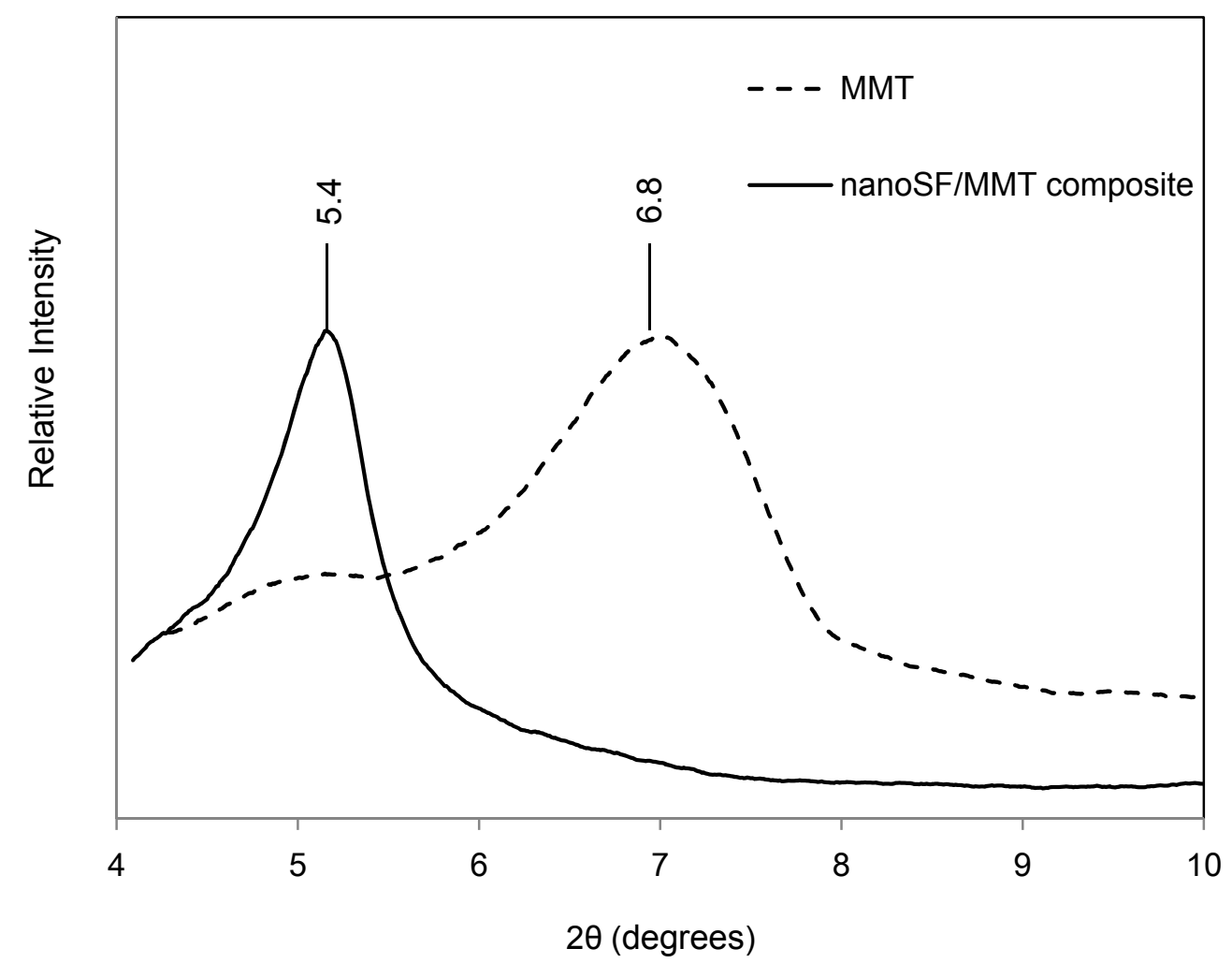

Nanocomposite of silk fibroin nanofiber and montmorillonite: fabrication and morphology

Yuki Kishimoto, Fuyu Ito, Hisanao Usami, Eiji Togawa, Masuhiro Tsukada, Hideaki Morikawa, Shigeru Yamanaka 


\section{Supplementary Data}

\section{Nanocomposite of silk fibroin nanofiber and montmorillonite: fabrication and morphology}

Yuki Kishimoto $^{\mathrm{a}}$, Fuyu Ito ${ }^{\mathrm{b}}$, Hisanao Usami ${ }^{\mathrm{a}}$, Eiji Togawa ${ }^{\mathrm{c}}$, Masuhiro Tsukada ${ }^{\mathrm{a}}$, Hideaki Morikawa ${ }^{\mathrm{a}}$, Shigeru

Yamanaka $^{\mathrm{a}}$

aFaculty of Textile Science and Technology, Shinshu University, 3-15-1 Tokida, Ueda, Nagano 386-8567, Japan

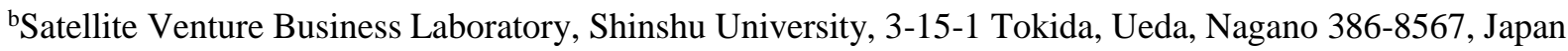

${ }^{c}$ Forestry and Forest Products Research Institute, 1 Matsunosato, Tsukuba 305-8687, Japan

FTIR analysis of nanosilk/MMT composite.

FTIR-ATR spectra for methanol-treated SF nanofiber, pure MMT, and nanoSF/MMT composite are shown in

Fig. S1. The FTIR spectra contained peaks from pristine MMT at $916 \mathrm{~cm}^{-1}, 1622 \mathrm{~cm}^{-1}$, and $3616 \mathrm{~cm}^{-1}$ and from methanol-treated SF nanofibers at $1516 \mathrm{~cm}^{-1}, 1622 \mathrm{~cm}^{-1}$, and $3280 \mathrm{~cm}^{-1}$. In the infrared spectra of the nanoSF/MMT composite, an absorption peak appeared at $1014 \mathrm{~cm}^{-1}$. This peak was not present in SF nanofibers, MMT or water. This peak was investigated for SF nanofiber plus MMT and MMT plus water systems.

The FTIR-ATR method was applied for the analysis. An IR spectrum of SF nanofibers plus MMT contained a peak at $975 \mathrm{~cm}^{-1}$, similar to that for MMT. Reactions between MMT and water were examined by dropping a mixture of MMT and water onto a prism and leaving it until the water evaporated. In the infrared spectrum (Fig. S2), the peak at $1012 \mathrm{~cm}^{-1}$ shifted to $975 \mathrm{~cm}^{-1}$ as the concentration of MMT increased due to water evaporation over time. These results confirm that the FTIR peak from the nanoSF/MMT composite at $1014 \mathrm{~cm}^{-1}$ does not indicate a reaction between MMT and the SF nanofiber, but a reaction between MMT and water. 


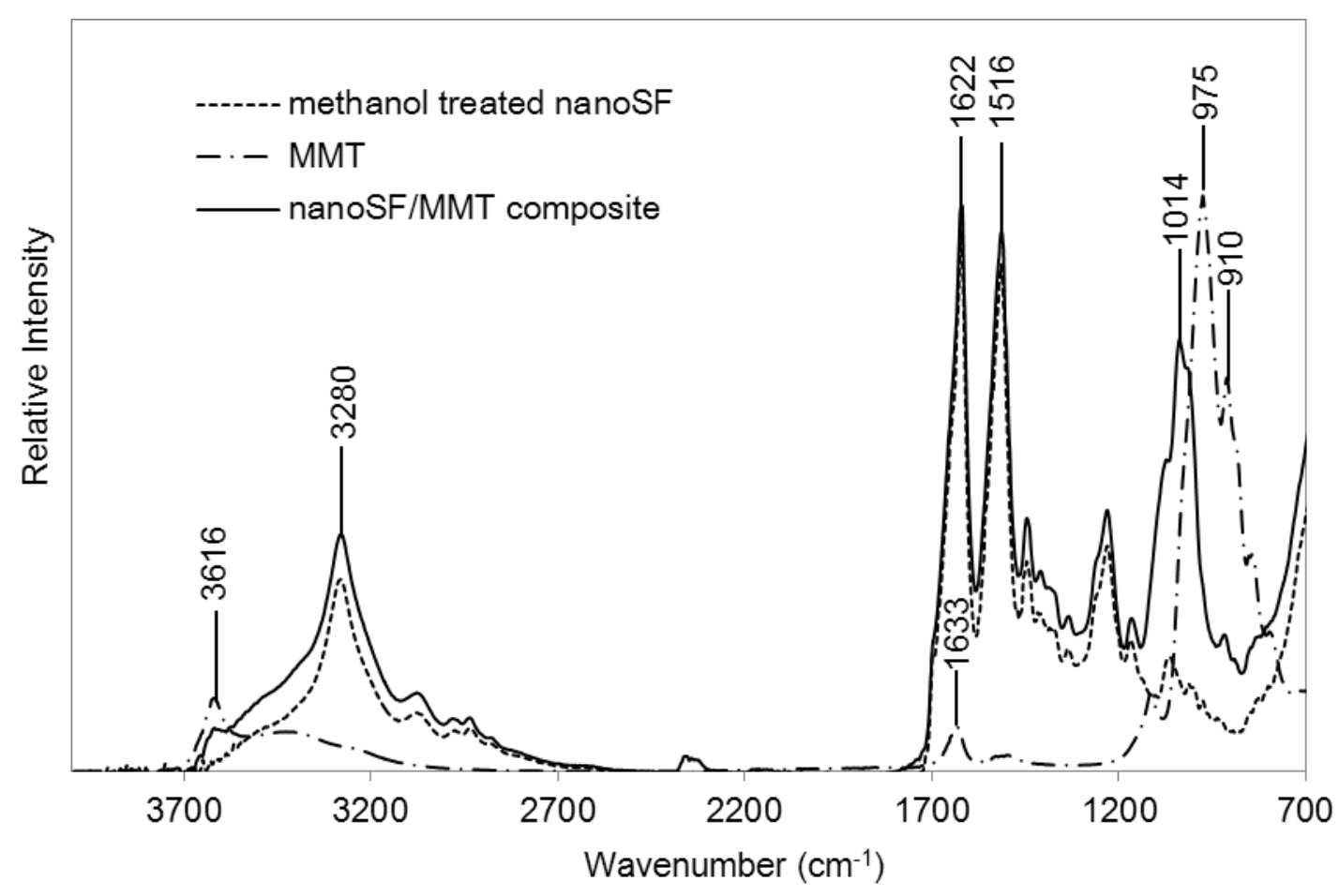

Fig. S1 FT-IR-ATR spectra for methanol treated nanoSF (dashed line), pure MMT (dashdotted line), and nano SF/MMT composite (solid line).

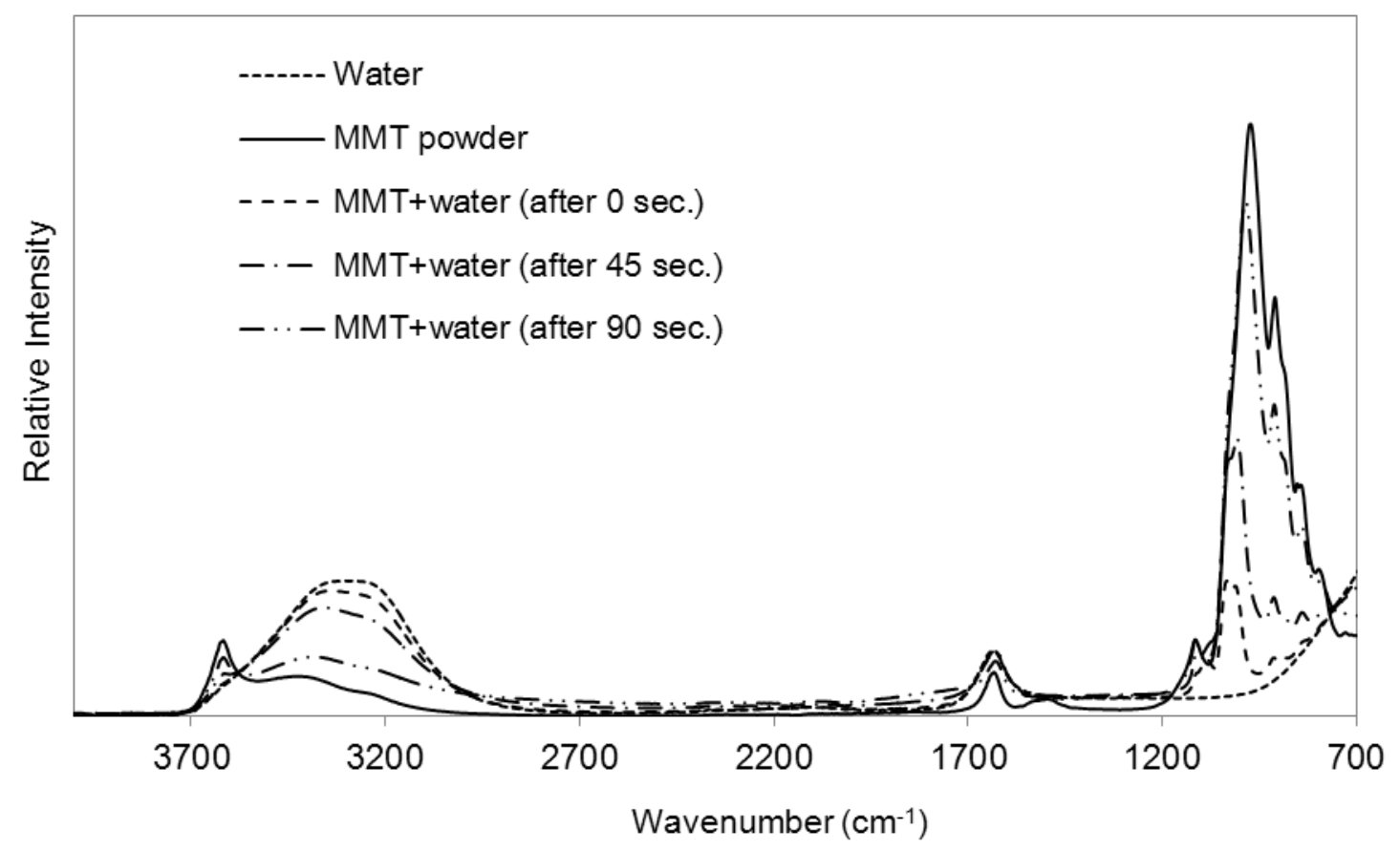

Fig. S2 FTIR-ATR spectra of MMT in water as the MMT concentration changed due to the evaporation of water from the sample. 


\section{Solid-state NMR analysis of composite.}

The spectra of SF and SF/MMT composite are shown in Fig. S3. Fig. S3 reveals that the ${ }^{13} \mathrm{C}$ NMR spectrum of SF/MMT composite is almost the same as that of the SF. This suggests that there is no formation of strong bonding between SF molecules and MMT.

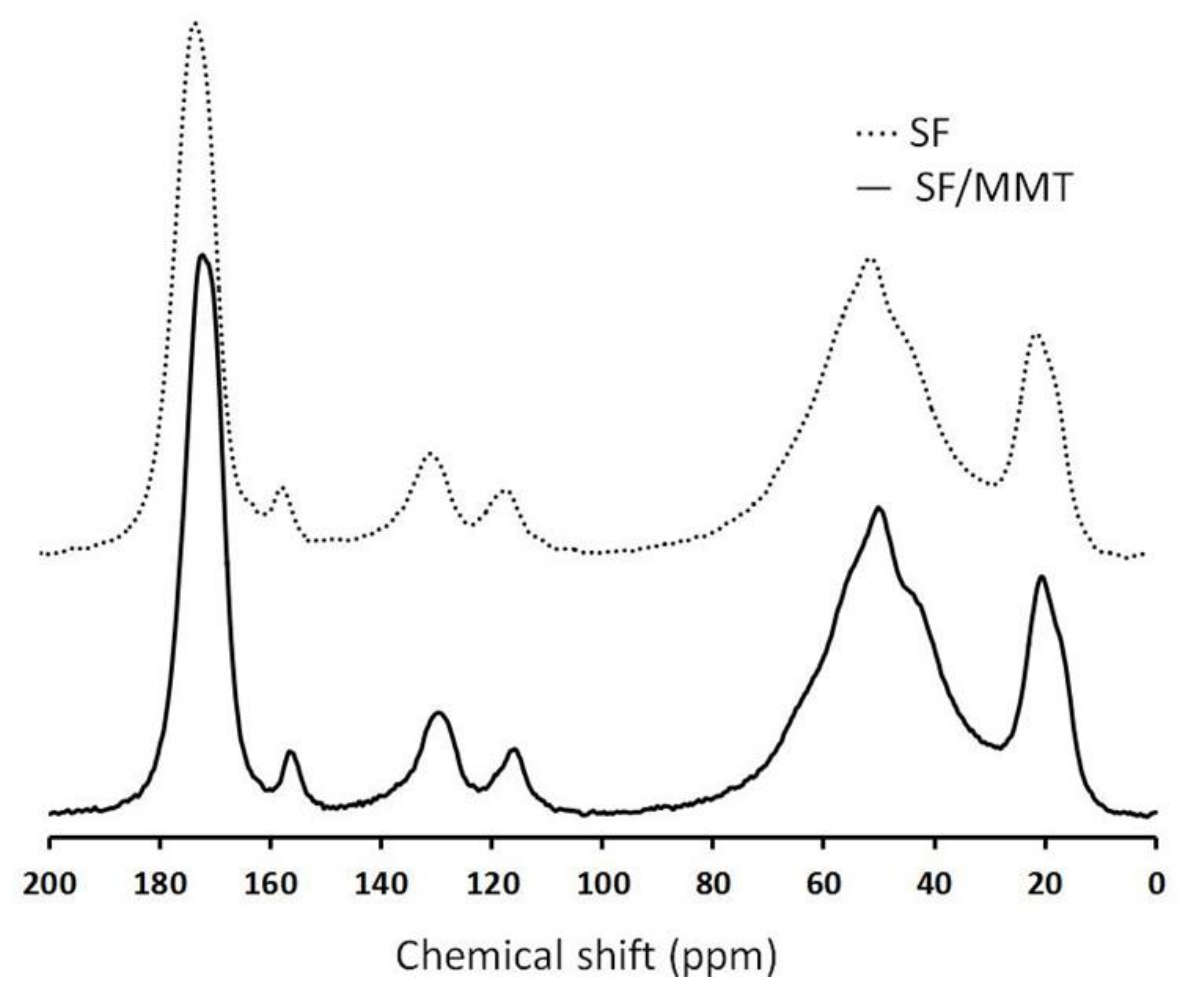

Fig. S3 ${ }^{13} \mathrm{C}$ NMR spectra of SF and SF/MMT composite. 